International Journal of Pediatric Otorhinolaryngology 32 (Suppl.) (1995) S45-S52

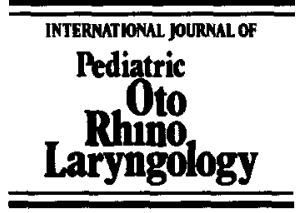

Symposium

\title{
The nose in children with unilateral cleft lip and palate
}

\author{
C.D.A. Verwoerd*a (Chairman), R. Mladina ${ }^{b}$, G.J. Nolst Trenitéc, \\ R.W. Pigott ${ }^{\mathrm{d}}$ \\ aDepartment of Otorhinolaryngology, Erasmus University Rotterdam/Sophia Children's Hospital \\ Rotterdam, Dr. Molewaterplein 40,3015 GD Rotterdam, The Netherlands \\ ${ }^{b}$ E.N.T. Department, University Hospital Salata, Zagreb, Croatia \\ 'Department of Otorhinolaryngology, Academic Medical Center, University of Amsterdam, \\ Amsterdam, The Netherlands

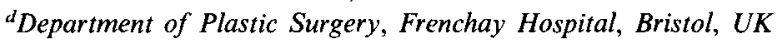

\begin{abstract}
Surgeons and orthodontists are still challenged to achieve 'better' noses for children with a unilateral cleft or lip, alveolus and palate (UCLP). Various aspects are discussed: infant anatomy and later changes, developmental mechanics, cleft syndrome in animals with surgically produced facial clefts, untreated patients with congenital clefts, the radical primary correction of the UCLP nose, the unsolved problems in secondary rhinoplasty and suggestions for scientific communication.
\end{abstract}

Keywords: Nose; Facial clefts; Facial development

\section{Developmental anatomy}

The changes of the facial profile during childhood and adolescence include an increasing anterocaudal prominence of the nose and maxilla. The accompanying structural changes of the nasal skeleton, are not all mapped out yet. The infant nose demonstrates specific anatomical features, not present in adulthood [7,17]. In the

\footnotetext{
* Corresponding author, Fax: 31104633102.
} 
newborn, the septodorsal cartilage is the only supporting structure of the nose. The mechanical stability of the structure is provided by the T-bar configuration of this complex cartilage (septum + upper laterals). The septal part reaches from the sphenoid to the columella and is firmly attached to the anterior nasal spine. The laterodorsal extensions form the cartilaginous nasal dorsum, and extend under the nasal bones to merge with the equally cartilaginous central part of the anterior cranial base [8]. Actually, the septodorsal cartilage is a prolongation of the cranial base. In the neonate, the vomer is the only osseous part of the septum. It is represented by a thin lamella between the basal rim of the cartilaginous septum and the palates and on both sides covered by nasal mucosa [21].

Postnatal development comprises (a) growth and (b) restructuring and remodelling of the anatomical components of the nose. The septum shows a progressive enchondral ossification [20,21], resulting in the formation of the perpendicular plate, which will fuse with the vomer between 5 and 7 years of age. The dorsolateral parts of the septodorsal cartilage demonstrate a progressive regression in craniocaudal direction until the proportionally much smaller 'adult' triangular cartilages remain with only a minor overlap from the nasal bones. At present, data are available for the neonatal and adult stage, but further observations at intervening ages should be most welcome.

\section{Developmental mechanics}

Also, the 'mechanics' of nasal and facial development are not yet fully explored. Animal experiments have produced evidence for an important role of the septodorsal cartilage with respect to the normal growth of the nasal bones, (pre)maxilla and orbit, which are responsible for the postnatal change of facial profile. Growth (lengthening) in sagittal direction of a thickening basal zone in the septal cartilage (from sphenoid to premaxilla) was demonstrated to enhance, via the attachment to the anterior nasal spine, the sagittal growth and anterocaudal displacement of the maxilla in the postnatal period. The sagittal growth of the nasal dorsum depends primarily on sagittal expansion of another zone of thick septal cartilage, extending from the sphenoid to the nasal dorsum $[22,23]$.

Symmetrical support by the upper lateral cartilages is essential to ensure the straight outgrowth of the nose and to avoid asymmetry of the nasal dorsum [23,26]. The nasal bones are passive, as far as morphogenesis is concerned. Their form is determined by the underlying septodorsal cartilage.

A balanced and combined action of the growing septodorsal cartilage on one side, and of the maxilla, vomer and nasal bones on the other side, is essential for the postnatal development of the face. Facial clefts including alveolus and palate will disturb this three-dimensional balance.

This is clearly demonstrated by surgically produced unilateral clefts of alveolus and palate in 4-week-old rabbits, which affect the further development of the nose and (pre)maxilla up to the adult stage (24 weeks after birth).

During growth the lengthening basal rim of the septal cartilage and the connecting half of the upper jaw deviate to the non-cleft side and reach a normal sagittal 
position and dimension. The maxillary fragment on the cleft side does not show the normal displacement in anterior direction during the postnatal period, because it lacks the forward 'pull' of the nasal septum. The result is a retroposition of the maxilla on the cleft side in the adult stage. Moreover, the maxilla on the cleft side shows a variable collapse to the midline. The nasal dorsum and caudal part of the septum are deviated to the non-cleft side [24].

\section{Untreated patients}

How can this experimentally induced 'cleft syndrome' be compared to findings in untreated patients with an unilateral cleft lip, alveolus and palate (UCLP)? Skulls with a unilateral cleft of alveolus and palate, dating from a pre-treatment period [6] showed similar characteristics as observed in the animal experiments: a deviation of the upper jaw to the non-cleft side and a retroposition with collapse of the maxillary fragment on the cleft side. These skulls further showed a specific malformation of the nasal septum: (a) deviation of the basal rim of the septal cartilage to the cleft side and (b) disjunction between the septal cartilage and the perpendicular plate and vomer. Actually, the latter, only connected to the palate on the non-cleft side, tends to a more horizontal position, which adds to the impression of a broadened nasal floor on the non-cleft side [21].

On lateral cephalograms, in untreated UCLP patients with superimposed images of cleft- and non-cleft side, a normal size and sagittal position of the maxilla is generally observed [1]. This probably reflects the situation on the non-cleft side. Some authors have described a retroposition of the posterior border of the maxilla, which could refer to the cleft side [18]. Significant deformations of the dental arch are mostly found in the maxilla of unilateral UCLP patients [9]. Although exact descriptions are not available, the length and prominence of the nose in adult non-treated patients with UCLP do not seem abnormal [14].

The similarities between the 'cleft syndrome' developed in animals with surgically produced clefts and in patients with non-treated congenital clefts, suggest that also in children the cleft is the cause of an abnormal pattern of growth, resulting in malposition and malformation of the facial components. In theory prevention of such an abnormal growth pattern should be possible.

\section{The secondary cleft nose deformity}

Although the results of orthodontic and surgical treatment of facial clefts are gradually improving, the secondary cleft nose in UCLP patients often demonstrates some or all of the following features $[4,19]$ :

1. asymmetry of the tip, columella, nostril, ala and nostril floor;

2. deflection of the caudal part of the septum to the non-cleft side;

3. stenosis of the vestibule on the cleft side;

4. hypoplasia of the maxilla beneath the alar attachment on the cleft side and variable collapse of the maxilla with asymmetry of the piriform aperture; 
5. underdevelopment of the maxilla on both sides with retroposition of the anterior nasal spine;

6. deviation of the cartilaginous and bony nasal dorsum;

7. deviation of the posterior part of the nasal septum to the cleft side.

The secondary cleft nose is often functionally insufficient: blocked to nasal breathing, suboptimal speech and recurrent infections.

Some of the above-mentioned features i.e. 4,6 and 7 were also encountered in untreated patients. The others seem to be related to and the result of the surgical closing procedure of the cleft.

Mladina reported data on septal deformities in 'normal' children, and on the association of septal deviation and facial clefts [11]. Septal deformities were present in $27.4 \%$ of the 923 control children. Vertical septal deflections in the valve area were predominant. Septal deviation with septovomeral or ethmoidovomeral disjunction occurred in $9 \%$. The latter type of septal deformity was found in $75 \%$ of the UCLP $(n=43)$ children.

For several years, it was advocated not to touch the nose during primary cleft lip surgery because of the risk of severe disturbance of the later nasal development. Animal experiments demonstrated that resection of the anterior part of the thickened basal rim of the septal cartilage (equally present in animals and human neonates) should be avoided because of the risk of maxillary underdevelopment. On the other hand, no arguments can be derived from animal experiments against repositioning the alar cartilages or even the septodorsal cartilage on condition that damage to the perichondrium, and fracturing or local necrosis of cartilage are avoided. An essential point is the limited wound healing capacity of cartilage. Fracturing or transection are not followed by complete regeneration and 'weak lines' in the cartilage remain which give rise to angulation or duplicature during further growth [25]. The external underdevelopment of the maxilla, occasionally observed in UCLP, could be caused by partial loss of the basal rim of the cartilaginous septum, injured during the primary cleft surgery. Necrosis of cartilage leads to formation of scar tissue, which interferes with normal growth [25].

Prevention (to some degree) of the secondary cleft nose deformity is a challenge, widely held [10]. An interesting approach is the repositioning of the ala on the cleft side, combined with the primary lip repair as discussed by Pigott in the next section.

\section{Radical primary correction of the unilateral complete cleft lip nose}

Alar leapfrog [15], the radical primary correction of the unilateral cleft lip nose starts with advancing the lateral crus of the alar cartilage along an intercartilaginous incision for $3-5 \mathrm{~mm}$, and the medial crus along a membranous septal incision for $2-3 \mathrm{~mm}$, depending on the severity of the congenital displacement. The next step is locking them in this position with a suture of 6.0 chromic catgut which picks up the cut mucosa of the distal side of each limb of the V-shaped incision to suture them to each other and to the junction of the septal cartilage and tip of the upper lateral cartilage. As the suture is tightened, the $\mathrm{V}$ forms a $\mathrm{Y}$ and the flattened hoop of the alar cartilage reforms an apex comparable to the normal side. A 6.0 
chromic catgut suture is strong enough to hold the reconstruction in a 3-month-old baby only if radical undermining of the cartilage from overlying skin is performed laterally to the infra-orbital foramen, up to the nasal bones and across the contralateral dome. The intercartilaginous and membranous septal incisions are closed diagonally, thereby reinforcing the advancement. Additional mucosa is added to the nostril using mucosa from the medial lip element based on the vomerine flap, used to form the nostril floor through the alveolus.

The technique has been used consistently since 1972 and 20 years later it can be said that:

1. radical undermining of the skin over the nasal cartilages of 3-month-old infants does not impair growth of the nose tip;

2. good symmetry of the alar domes and nostril rims has been maintained in the majority of cases with no further intervention;

3. most of the children have suffered some degree of reduction of the circumference of the vestibule but this has appeared to be asymptomatic in the majority.

It is concluded that alar leapfrog reposition of the alar cartilage provides good support for the alar dome with the probability of reduced teasing about appearance.

\section{Rhinoplasty for correction of secondary cleft nose deformity}

Rhinoplasty for cleft nose deformity is a complex procedure including septal surgery, osteocartilaginous vault surgery, maxillary augmentation and vestibuloplasty with alar base reallocation. The choice of the surgical approach, external or endonasal, depends on the severity of the deformity. If there is a mild deformity of the nasal tip, a closed (endonasal) approach is appropriate in most cases. Nevertheless, the external approach is the approach of choice.

To rebuild a nose the following grafts and implants are often indicated: columella strut for tip projection, shield graft/tip graft for tip definition, dorsal graft for dorsal height, premaxillary implant to restore maxillary support for the nose (ala).

Autogenous septal or ear cartilage grafts are the first choice over allogenic and non-biological (xenogenic) material. For maxillary augmentation xenogenic material such as proplast and gore-tex can also be used. In severe retroposition the maxillary advancement procedure is indicated.

Nolst Trenité reviewed the results of 52 rhinoplasties, performed in cleft lip patients, between 1988 and 1993, and two specific problems were encountered. The first was a slight to moderate recurrence of the caudal septal deviation in nearly $30 \%$ of the cases. This problem diminished to a large degree after using a fixation suture around the reallocated anterior nasal spine to ensure the midline position of the caudal septum. The second problem appeared to be the recurrence of the postoperative narrowing of the vestibulum after vestibuloplasty. In $10 \%$ of the cases the postoperative situation was even worse than the pre-operative, probably due to retraction of scar tissue. To prevent scar tissue retraction, a special custom made device [13] was used based on the nostril splint, described by Nakajima et al. [12]. The patient has to wear this vestibulum device day and night during the first 6 
weeks after surgery and the following 6 weeks only at night. The preliminary results are encouraging.

\section{Communication of results}

The literature is replete with articles giving techniques for primary or secondary correction of the nose. Unfortunately, there are very few presenting adequate information. The following points may be considered.

1. Some reports only highlight 'best cases'. Actually, the cases reported should ideally be sequential with no omissions. The overall numbers of cases of tightly specified type and age, and of declared male-female ratio should be provided.

2. Cases of complete alveolar clefts are mixed in with total bony clefts (UCLP) but have very much better facial growth and bony support to the nose. Even with complete bony clefts the restraint of a Simonart's band prevents the worst degree of dislocation of the cartilage.

3. Statements like good, fair and unsatisfactory are not adequate vehicles for scientific communication. Photographic reporting of the outcome should be advocated and standardised. A few panel assessments of populations of cleft children have been made [3]. Since it is now possible to provide a numerical expression of symmetry [2,5] comparison of large numbers of patients treated with different techniques can be expected.

4. Reports should also present data pertinent to the inside anatomy of the nose and combine morphological features with functional measurements (patency of the nasal fossa, respiration, speech).

5. The articles should give a long term follow-up; for children the follow-up should include the adolescent growth spurt.

\section{Final remarks}

As far as the nose is concerned, the results - morphological and functional of current treatment of UCLP must be further improved. Clinical and experimental research should produce a rational base for a new type of surgery, growth regulating surgery, next to functional and aesthetic surgery. Both in primary and secondary surgery, the nasal vestibule on the cleft side remains a difficult problem, waiting for innovative management. As age-related differences in wound healing have been reported, an experimental study of wound healing of facial structures in very young animals could give clues to the optimal age for primary surgery. Other developments could be in situ stimulation of wound healing and the use of active implants, as demineralized bone matrix, which is converted into cartilage by colonization from a connected perichondrial flap [16].

Assessment of outcomes in the management of cleft lip and palate requires the sustained interest of a multidisciplinary team over periods longer than most professional careers. Every patient lost to follow-up from the primary management team, further extends and impairs the audit of outcome. The occasional perfor- 
mance of surgery outside and without discussion of the overall plan of management weakens the effort to improve understanding and management of this complex problem.

\section{References}

[1] Capelozza, L.C., Taniguchi, S.M. and da Silva, O.G. (1993) Craniofacial morphology of adult unoperated complete unilateral cleft lip and palate patients. Cleft Palate Craniofac. J. 30, 376-381.

[2] Coghlan, B.A., Laitung, J.K.G. and Pigott, R.W. (1993) A computer aided method of measuring nasal symmetry in the cleft lip nose. Brit. J. Plast. Surg. 46, 13-17.

[3] Cussons, P.D., Murison, M.S.C., Fernandez, A.E.L. and Pigott, R.W. (1993) A panel based assessment of early versus no nasal correction of the cleft lip nose. Brit. J. Plast. Surg. 46, 7-12.

[4] Farrior, T.F. (1993) The cleft lip nose: an update. Fac. Plast. Surg. 9, 241-268.

[5] Laitung, J.K.G., Coghlan, B.A. and Pigott, R.W. (1993) A comparison of computer versus panel assessment of two groups of patients with cleft lip and palate. Brit. J. Plast. Surg. 46, 18-21.

[6] Limborgh, J. van (1964) Some aspects of the development of the cleft-affected face. In: Hotz, R. (Ed.), Early Treatment of Cleft Lip and Palate. Huber, Bern, pp. 25-30.

[7] Loosen, J. van, Verwoerd-Verhoef, H.L. and Verwoerd, C.D.A. (1988) The nasal septal cartilage in the newborn. Rhinology 26, 161-166.

[8] Loosen, J. van, Klooswijk, A.J.J., Velzen, D. van and Verwoerd, C.D.A. (1990) Computed tomography of the human developing anterior skull base. Eur. J. Radiol. 10, 211-214.

[9] Mars, M. and Houston, W.J.B. (1990) A preliminary study of facial growth and morphology in unoperated male unilateral cleft lip and palate subjects over 13 years of age. Cleft Palate J. 27, $7-10$.

[10] McComb, H. (1985) Primary correction of unilateral cleft lip nasal deformity: a 10 year review. Plast. Reconstr. Surg. 75, $791-799$.

[11] Mladina, R. (1987) The role of maxillar morphology in the development of pathological septal deformities. Rhinology 25, 199-205.

[12] Nakajima, T., Yoshimura, Y. and Sakakibara, A. (1990) Augmentation of the nostril splint for retaining the corrected contour of the cleft lip nose. Plast. Reconstr. Surg. 85, 182-186.

[13] Nolst Trenité, G.J. (1994) Rhinoplasty in the cleft lip patient. Abstract book. VIth International Congress of Pediatric Otorhinolaryngology.

[14] Ortiz-Monasterio, F., Serrano, A.R., Barrera, G.P., Rodriguez-Hoffman, H. and Vinageras, E. (1966) A study of untreated adult cleft palate patients. Plast. Reconstr. Surg. 38, 36-41.

[15] Pigott, R.W. (1985) Alar leapfrog: a technique for repositioning the total alar cartilage at primary cleft lip repair. In: Saunders, W.C. (Ed.), Clinics in Plastic Surgery. Cleft Lip and Palate, Trier, Philadelphia, 12, 643-658.

[16] Pirsig, W., Bean, J.K., Lenders, H., Verwoerd, C.D.A. and Verwoerd-Verhoef, H.L. (1995) Cartilage transformation in a composite graft of demineralized bovine bone matrix and ear perichondrium used in a child for the reconstrucltion of the nasal septum. Int. J. Ped. Otorhinolaryngol. (in press).

[17] Poublon, R.M.L., Verwoerd, C.D.A. and Verwoerd-Verhoef, H.L. (1990) Anatomy of the upper lateral cartilages in the human newborn. Rhinology $28,41-45$.

[18] Ross, R.B. (1987) Treatment variables affecting facial growth in complete unilateral cleft lip and palate. Part 1. Treatment affecting growth. Cleft Palate J. 24, 5-23.

[19] Salyer, K.E. (1992) Early and later treatment of unilateral cleft nasal deformity. Cleft Palate Craniofac. J. 29, 556-569.

[20] Schultz-Coulon, H.L. and Eckermeier, L. (1976) Zum postnatalen Wachstum der Nasenscheidenwand. Acta Otolaryngol. (Stockh.) 82, 131-142.

[21] Verwoerd, C.D.A., Loosen, J. van, Schütte, H.E., Verwoerd-Verhoef, H.L. and Velzen, D. van (1989) Surgical aspects of the anatomy of the vomer in children and adults. Rhinology Suppl. 9, $87-96$. 
[22] Verwoerd, C.D.A. and Verwoerd-Verhoef, H.L. (1989) Developmental aspects of the deviated nose. Facial Plast. Surg. 6, 95-100.

[23] Verwoerd, C.D.A. and Verwoerd-Verhoef, H.L. (1992) Rhinosurgery in children: surgical and developmental aspects. In: Nolst Trenité, G.J. (Ed.), Rhinoplasty: A Practical Guide to Functional and Aesthetic Surgery of the Nose. Kugler, Amsterdam, pp. 149-156.

[24] Verwoerd, C.D.A., Urbanus, N.A.M. and Verwoerd-Verhoef, H.L. (1979) Growth mechanisms of skulls with facial clefts. Acta Otolaryngol. 87, 335-339.

[25] Verwoerd, C.D.A., Meeuwis, C.A. and Verwoerd-Verhoef, H.L. (1989) Stress and wound healing of the cartilaginous nasal septum. Acta Otolaryngol. (Stockh.) 107, 441-445.

[26] Verwoerd-Verhoef, H.L. and Verwoerd, C.D.A. (1994) Sinonasal surgery and growth; an experimental study review. Proc. XVth ERS congress, Kopenhagen-Denmark (in press). 